\title{
LA MOTIVACIÓN SOSTENIDA EN EL PROCESO ENSEÑANZA APRENDIZAJE, UN DESAFÍO EN EL AULA DE CLASE
}

\author{
Mariella Victoria Mendoza Carrasco
}

\begin{abstract}
RESUMEN
El presente artículo es de naturaleza teórico, el cual permitirá a los docentes conocer la evolución en el concepto de la "Motivación escolar" y analizar e interpretar los diferentes factores que intervienen para mantener, una "motivación sostenida y continua" en el proceso de enseñanza - aprendizaje. A su vez, le permitirá conocer y utilizar técnicas, que posibiliten el interés y el deseo de aprender con esfuerzo y constancia; hacia el logro de fines apropiados y la realización de propósitos definidos como son; los logros de los objetivos trazados en las diferentes áreas pedagógicas.
\end{abstract}

El papel del docente es inducir motivos en sus alumnos, para despertar el interés durante el proceso de los aprendizajes y comportamientos, y así llamar su atención, estimulando el deseo de aprender, manteniendo una motivación sostenida durante todo el proceso.

\section{PALABRAS CLAVE}

Motivación, interés, actitud, proceso, aprendizaje, logros.

\section{ABSTRACT}

This article is of a theoretical nature, which will allow teachers to know the evolution of the concept of "School Motivation" and to analyze and interpret the different factors that intervene to maintain a "sustained and continuous motivation" in the teaching process - learning. In turn, it will allow you to know and use techniques, which will enable the interest and desire to learn with effort and constancy; Towards the achievement of appropriate ends and the realization of definite purposes as they are; The achievements of the objectives drawn in the different pedagogical areas.

The role of the teacher is to induce motives in his students, to arouse interest during the process of learning and behavior, and thus attract their attention, stimulating the desire to learn, maintaining a sustained motivation throughout the process.

\section{KEYWORDS}

Motivation, interest, attitude, process, learning, achievements.

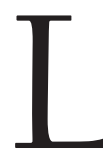

a motivación escolar es lo que induce y estimula al alumno a la voluntad de aprender, es el interés por su propio aprendizaje o por las actividades que le conducen a él; está compuesta de necesidades, deseos, tensiones, incomodidades y expectativas; es un proceso exclusivamente endógeno, interpersonal, donde intervienen de cierta manera los factores interpersonales o sociales. Entran en juego la afectividad, la razón, la voluntad, y la apetencia.

La motivación escolar es un factor cognitivo presente en todo acto y proceso de aprendizaje; condiciona la forma de pensar del alumno y con ello el tipo de aprendizaje resultante. Una buena motivación conlleva a la autoconfianza de la capacidad de poder lograr los objetivos del aprendizaje, esta no se restringe a la aplicación de una técnica o método de enseñanza en particular; por el contrario, la motivación escolar conlleva una compleja interrelación de diversos componentes cognitivos, afectivos, sociales y de carácter académico que se encuentran involucrados y que de una u otra forma tienen que ver con las actuaciones de los alumnos como la de sus profesores.

El interés se puede adquirir, mantener o aumentar en función de elementos intrínsecos y extrínsecos; sin embargo, hay que distinguir de lo que tradicionalmente se ha venido llamando en las aulas motivación, que no es más que lo que el profesor hace para que los 
alumnos se motiven. En la escuela tradicional llamábamos motivación, al proceso que se origina al inicio de una actividad o tarea del aprendizaje como punto de partida; sin embargo, vemos que la motivación se debe mantener dentro del proceso y hasta el final del aprendizaje, para dar nuevas motivaciones y expectativas dentro de ellos, que le permitan mejorar el rendimiento académico y el comportamiento disciplinario en los estudiantes. Se debe iniciar la motivación, mantenerla y dirigir la conducta motivadora de un individuo hacia la realización de la tarea y aprendizaje.

Cada alumno se motiva por razones diferentes, en la práctica se observa una limitada eficacia de las motivaciones colectivas. Podemos llegar a la conclusión que si no van las motivaciones acompañadas de una individualización y adecuación a las peculiaridades del alumno, no vamos a lograr una eficaz motivación intrínseca del estudiante en los efectos motivacionales dentro del proceso de enseñanza aprendizaje, sin dejar de considerar y mencionar que influyen tanto los rasgos de personalidad como la misma historia de cada alumno, la cual está determinada por su ambiente familiar o contexto socioeconómico y cultural.

La motivación como proceso auto energético de la persona, limita la función del profesor a ser un agente exterior que trata de desencadenar la motivación intrínseca del alumno. Esto nos lleva a una consecuencia: los incentivos tienen un valor motivacional limitado. La misma actividad incentivadora produce distintas respuestas en distintos individuos, o incluso en el mismo alumno en diversos momentos. La motivación condiciona la forma de pensar del alumno y con ello el tipo de aprendizaje resultante.

El mecanismo de la motivación se desarrolla en tres etapas:

a) Aprehensión de un valor para su vida y sus aspiraciones.

b) Los alumnos se convencen de que pueden conseguir ese valor.

c) Liberación del esfuerzo personal para conquistar el valor.

\section{$\varnothing$ Propósitos de la motivación escolar}

1. Despertar el interés en el alumno y dirigir su atención sostenida, durante el proceso de enseñanza-aprendizaje.

2. Estimular el deseo de aprender que conduce al esfuerzo y la constancia.

3. Dirigir estos intereses y esfuerzos hacia el logro de fines apropiados y la realización de propósitos definidos como son; los logros de los objetivos y metas trazadas.

\section{$\varnothing$ Tipos de motivación}

Positivas de dos clases:

a) Intrínseca: interés positivo manifestado como estado interno voluntario, la acción proviene de factores internos como lo son: valores, intereses, actitudes, expectativas, pensamientos, entre otros, que activa y dirige y mantiene la conducta atenta, placentera y con interés por la materia, siendo vital para el aprendizaje como en el campo de trabajo de tareas.

Para motivar intrínsecamente a los alumnos, se requiere lograr:

Que den más valor al hecho de aprender que al de tener éxito o fracaso.

Que consideren a la inteligencia y a las habilidades de estudio como algo modificable, y no como inmutables.

Que centren más su atención en la experiencia de aprender que en las recompensas externas.

Son dos las condiciones que deben darse para que se produzca en un individuo la motivación intrínseca hacia la realización de una tarea:

Que la realización de la tarea sea ocasión para percibir o experimentar que se es competente.

Que se dé la experiencia de autonomía, que el sujeto sienta que ejerce control sobre su entorno y su propia conducta. 
b) Extrínseca: interés resultante, cuando la fuente de la motivación es un elemento ambiental externo al sujeto que la muestra.

Está presente cuando la acción a realizar tiene un fin propio, buscando que el resultado tenga como recompensa un resultado positivo, como premio o para evitar un castigo.

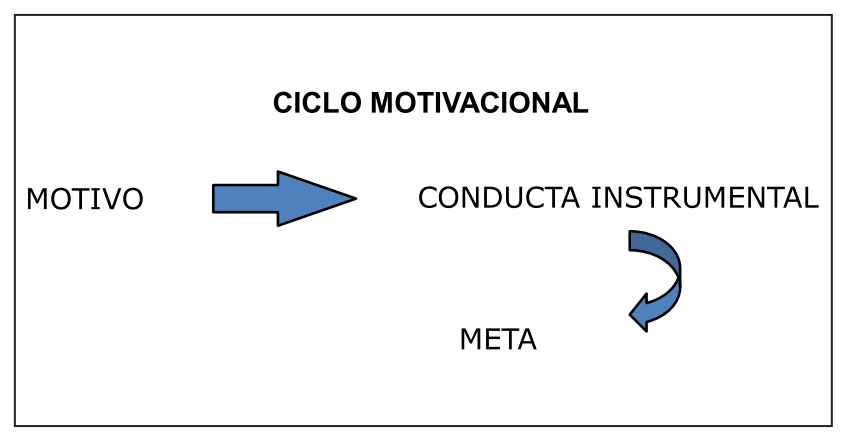

Una de las teorías motivacionales de corte humanista más renombrada es "La teoría motivacional" de Abraham Maslow. La motivación humana está determinada por la jerarquía de necesidades.

Maslow llegó a la conclusión de que la autorrealización es un proceso continuo y no un producto acabado; además, consideró equivalentes a la autorrealización con la palabra sanidad, concluyendo que las personas sanas poseen ciertas características:

Manifiestan eficaz percepción de la realidad y aceptación a ella.

Se aceptan a símismos y a otros.

Muestran elevado grado de espontaneidad.

A veces sienten la necesidad de soledad y aislamiento.

Son autónomos o bastante independientes con sus ambientes.

Cultivan profundas relaciones interpersonales con otros.

Democráticos.

Creativos.

Tienden a ser inconformistas.

Son autónomos o bastante independientes con sus ambientes. $\varnothing$ ¿Qué rol cumple el profesor en la motivación?

El papel del docente es inducir motivos en sus alumnos para despertar el interés durante el proceso de los aprendizajes y comportamientos, y así llamar su atención estimulando el deseo de aprender y poder aplicarlo de manera voluntaria al realizar satisfactoriamente los trabajos de clase, dirigiendo estos intereses y esfuerzos hacia el logro de los objetivos. La ausencia de motivación hace complicada la tarea del profesor; sin motivación no hay un eficaz y eficiente aprendizaje.

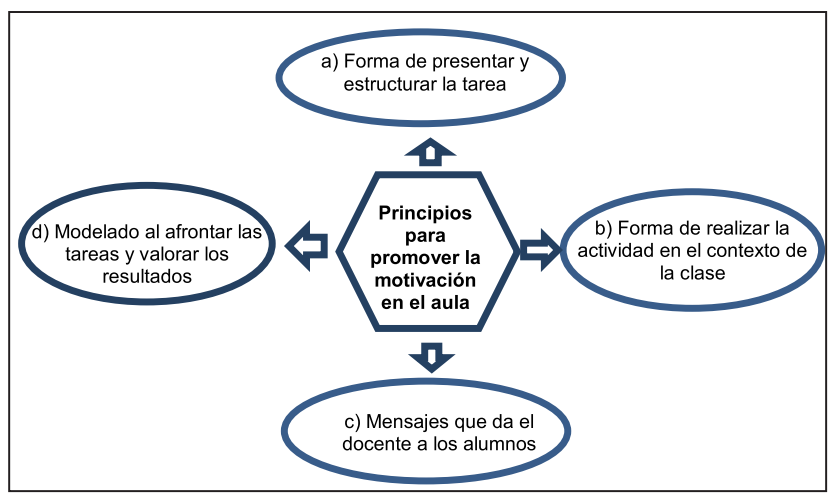

Los factores que determinan la motivación en el aula se dan a través de la positiva interacción entre el profesor y el alumno, desde este punto de vista, el profesor debe plantearse un triple objetivo en su acción motivadora:

Suscitar elinterés.

Dirigiry mantener el esfuerzo.

Lograr el objetivo de aprendizaje prefijado.

El entusiasmo del profesor, el clima que reina en la clase, las buenas relaciones entre los miembros, alumnos y profesor o entre los mismos alumnos, influye en la motivación; para ello hay que apoyarse en los intereses de los alumnos y conectarlos con los objetivos del aprendizaje o con la misma actividad. Un factor importante es disponer de una buena planificación del proceso enseñanza aprendizaje y tener un buen sistema de recompensa o premio de sus logros para los alumnos. 
Los buenos estudiantes están motivados por el aprendizaje en sí mismos, los malos estudiantes muchas veces, por las recompensas externas que pueden obtener, considerando que cada alumno responde de manera diferente. Hay muchos profesores que tienden a buscar técnicas interesantes para ellos, pero que no provocan ninguna motivación en el alumno. Los alumnos no se motivan por igual, por lo que es importante buscar y realizar actividades motivadoras variadas, que impliquen mayor participación e interés de los alumnos de manera sostenida.

\begin{tabular}{|l|l|}
\hline \multicolumn{2}{|c|}{ Factores involucrados en la motivación } \\
\hline ALUMNO & Tipo de metas que establecen. \\
& Expectativas de logro. \\
& Atribuciones de éxito y fracaso. \\
& Habilidades de estudio, planeación, automonitoreo. \\
& Manejo de la ansiedad y el estrés. \\
& Autoeficacia percibida. \\
\hline PROFESOR & Actuación pedagógica (orientación al aprendizaje \\
& significativo). \\
& Manejo interpersonal. \\
& Mensajes y retroalimentación a los alumnos. \\
& Expectativas y representaciones (pensamiento, \\
& creencias). \\
& Organización social de la clase (estructuras de \\
& aprendizaje). \\
& Formas en que recompensa y sanciona; evaluación. \\
& Comportamientos que modela. \\
\hline CONTEXTOE & Valores y prácticas de la comunidad educativa. \\
INSTRUCCIÓN & Clima de aula. \\
& Proyecto educativo y currículo. \\
& Influencias familiares, culturales, de los pares. \\
\hline
\end{tabular}

Los comportamientos y valores que el profesor modela en los alumnos son de gran importancia, ya que pueden facilitar o inhibir el interés de éstos por el aprendizaje.

El ambiente o clima motivacional que prima en el aula y el empleo de una serie de principios motivacionales que el docente utiliza en el diseño y conducción del proceso de enseñanza aprendizaje, son factores que intervienen en la motivación sostenida.

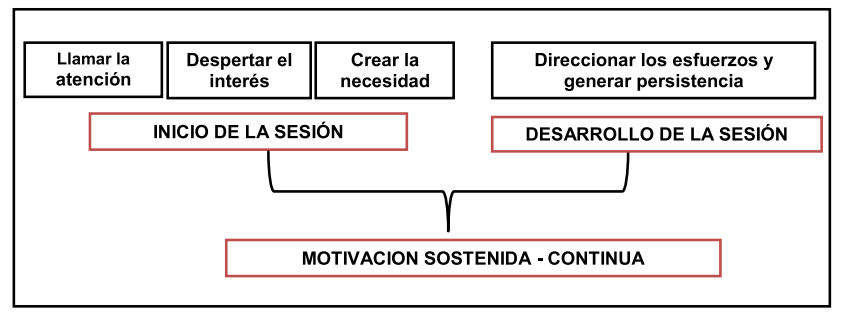

\section{$\varnothing \quad$ ¿Cómo motivar a un alumno?}

Para saber cómo motivar, se debe tener en cuenta algunos de los siguientes aspectos:

Explicar a los alumnos los objetivos educativos que tenemos previstos para la determinada sesión.

Justificar la utilización de los conocimientos que les intentamos transmitir con las actividades que les vamos a plantear.

Plantearles las actividades de forma lógica yordenada.

Proponerles actividades que les hagan utilizar distintas capacidades de su resolución.

Fomentar la comunicación entre los alumnos y las buenas relaciones, realizando tareas de grupo.

Plantear el razonamiento y la comprensión como la mejor herramienta para la resolución de actividades y conflictos.

Tomar los errores como nuevos momentos de aprendizaje y como momentos enriquecedores. Fomentar la comunicación entre los alumnos y las buenas relaciones, realizando tareas de grupo. Plantear el razonamiento y la comprensión como la mejor herramienta para la resolución de actividades y conflictos.

Aplicar los contenidos y conocimientos adquiridos a situaciones próximas y cercanas para los alumnos.

\section{$\varnothing \quad$ La motivación escolar y sus efectos en el aprendizaje}

La motivación en el aula es un proceso general y prolongado, este proceso involucra variables tanto cognitivas como afectivas; cognitivas en cuanto a las habilidades de pensamiento y conductas instrumentales para alcanzar las metas propuestas; afectivas, en tanto que comprende elementos como la autovaloración, y autoconcepto. Ambas variables actúan en interacción a fin de complementarse y hacer eficiente la motivación, proceso esencial e 
importante dentro del proceso de enseñanza y Los factores que determinan la motivación en el aprendizaje. La motivación escolar no es una técnica o método de enseñanza particular, sino un factor cognitivo presente en todo acto y proceso de aprendizaje. aula se dan a través de la positiva interacción entre el profesor y el alumno. Hay metas que logra el alumno a través de la actividad escolar y, a su vez, genera la satisfacción personal y la autovaloración de su desempeño.

\section{REFERENCIAS}

Alonso Tapia, J. (1997). Motivar para el aprendizaje. España-Barcelona.

Díaz Barriga Arceo, Frida y Hernández Rojas, Gerardo (1998). Estrategias docentes para un aprendizaje significativo. Una interpretación constructivista. México: Ed. Mc. Graw Hill.

Díaz, F.; Hernández, G. (1999). La motivación escolar y sus efectos en el aprendizaje. En: Estrategias docentes para un aprendizaje significativo. México: McGraw Hill.
J. A. (1996). "Motivación en el aula" y "Principios para la intervención motivacional en el aula". En: Motivación. Querer aprender. Argentina-Buenos Aires.

Juvonen, J. y Wentzel, K. R. (2001). Motivación y adaptaciónescolar. México: Oxford.

Wentzel, K. R. (2001). Metas sociales y relaciones sociales como motivadoras de la adaptación a la escuela. 